\title{
Is It Time to Beta Block the Septic Patient?
}

\author{
Philip Pemberton, ${ }^{1}$ Tonny Veenith, ${ }^{1,2,3}$ Catherine Snelson, ${ }^{1}$ and Tony Whitehouse ${ }^{1,4}$ \\ ${ }^{1}$ Department of Anaesthesia and Critical Care Medicine, University Hospitals Birmingham NHS Foundation Trust, \\ Queen Elizabeth Hospital Birmingham, Mindelsohn Way, Birmingham B15 2GW, UK \\ ${ }^{2}$ Department of Infection and Inflammation, University of Birmingham, Edgbaston, Birmingham B15 2TT, UK \\ ${ }^{3}$ Department of Medicine, University of Cambridge, The Old Schools, Trinity Lane, Cambridge CB2 1TN, UK \\ ${ }^{4}$ College of Medical and Dental Sciences, University of Birmingham, Edgbaston, Birmingham B15 2TT, UK \\ Correspondence should be addressed to Tony Whitehouse; tony.whitehouse@uhb.nhs.uk
}

Received 13 March 2015; Accepted 18 May 2015

Academic Editor: Elizabeth Papathanassoglou

Copyright (C) 2015 Philip Pemberton et al. This is an open access article distributed under the Creative Commons Attribution License, which permits unrestricted use, distribution, and reproduction in any medium, provided the original work is properly cited.

Beta blockers are some of the most studied drugs in the pharmacopoeia. They are already widely used in medicine for treating hypertension, chronic heart failure, tachyarrhythmias, and tremor. Whilst their use in the immediate perioperative patient has been questioned, the use of esmolol in the patients with established septic shock has been recently reported to have favourable outcomes. In this paper, we review the role of the adrenergic system in sepsis and the evidence for the use of beta stimulation and beta blockers from animal models to critically ill patients.

\section{Introduction}

Although the mortality from septic shock has fallen in recent years, this has been through improved detection and earlier antibiotic therapy. Despite intensive research over 20 years, interventions to alter the course of sepsis once the immune response has initiated have not been found. Recently, a single centre phase-II study from Italy [1] reported that beta-adrenergic blockade in patients with septic shock who continued to have elevated heart rates after standard fluid resuscitation caused improvements in cardiac performance, plasma lactate clearance, and a reduction in vasopressor dependence, with no reported adverse effects. The study with 77 patients in each group was not powered to examine survival but nevertheless showed substantial reduction in short-term mortality (adjusted hazard ratio, 0.39; 95\% CI, 0.26 to $0.59 ; P<0.001$; twenty-eight-day mortality was $49.4 \%$ in the esmolol group compared with $80.5 \%$ in the control group). This raises the question whether beta blockers could offer a new way of managing the critically ill patient with septic shock and if so how its benefits may arise.

In addition to the adrenoceptors found throughout the cardiovascular system, the adrenergic system is also a powerful modulator of the immune system [2]. Lymphoid organs (spleen, thymus, lymph nodes, and bone marrow) are predominantly innervated by the sympathetic system. With the exception of $\mathrm{T}$ helper type 2 (Th2) cells, the majority of lymphoid cells express beta-adrenergic receptors on their surface. The adrenergic system also modulates or regulates cell death, mitochondrial function, and inflammatory signalling [3]. Bone marrow production and differentiation of monocytes is influenced by the adrenergic system $[4,5]$ and immune cell apoptosis is at least partly mediated by catecholamines, via alpha-adrenergic and beta-adrenergic pathways. Although there has been a great deal of focus on the cardiovascular benefits of beta blockade in sepsis [6], the ubiquitous nature of the adrenergic system brings into question whether there are other mechanisms through which beta blockers may exert their influence.

\section{The (Patho)physiology of the Sympathetic System during Septic Shock}

Interaction between sympathetic nervous and immune systems is mediated with an effector arm consisting of catecholamines and inflammatory cytokines. In response to invading pathogens, there is up-regulation of sympathetic activity enabling the host to mount a rapid and effective 
response. It contributes to the early clinical presentation in sepsis of flushing, and tachycardia and hypotension caused, in part, by sympathetic mediated vasodilatation. That is to say that the cross-talk between the sympathetic system and the immune system is "physiological" rather than "pathological" in the first instance. However, there comes a point at which such an effector system begins to cause injury to the host [7]. The continued elevation of catecholamines observed in some septic patients becomes unfavourable and for some reason does not down regulate [8].

The pathophysiology of septic shock includes excessive sympathetic outflow and high concentrations of plasma catecholamines leading to vasodilatation followed by vasoconstriction, vascular hyporeactivity, myocardial depression, and autonomic dysfunction $[7,8]$. The recommended treatment for fluid-unresponsive sepsis-related hypotension is norepinephrine [9], but this agent has numerous adverse effects including direct myocardial damage, insulin resistance, thrombogenicity, immunosuppression, and enhanced bacterial growth [10]. This hypercatecholamine state is in part also responsible for numerous compensatory metabolic alterations characteristic of the stress condition, including the dysregulation of glycaemic control seen after injury and sepsis [11, 12]. Some of these adverse effects could be attenuated by beta-adrenergic blockade, since this enables heart rate control [13] and limits adverse events related to sympathetic overstimulation [10].

It was noted many years ago that epinephrine enhanced bacterial infections [14] and decreased the number of bacteria necessary for a lethal dose in both Clostridia species and pathogenic aerobic organisms. Catecholamines have been demonstrated to enhance biofilm formation and stimulate bacterial growth in Staphylococcus epidermidis [15]. Growth of Yersinia enterocolitica [16] is enhanced by dopamine and norepinephrine (but not ephedrine), an effect mediated by removal of iron from lactoferrin and transferrin by the catechol moiety and its subsequent acquisition by bacteria [17]. Escherichia coli O157:H7 and Salmonella enterica also have enhanced growth with catecholamines.

High plasma catecholamine levels have been noted in septic animals [18] and humans [8]. Boldt and colleagues observed elevated and persistently high plasma catecholamine levels in nonsurvivors in a critically ill patient population many of whom also received catecholamines as part of their treatment [19]. In septic shock patients, arterial norepinephrine levels are significantly associated with a greater mortality [8]. The extent and duration of catecholamine therapy and tachycardia are all independently associated with poor outcomes in critically ill patients [8, 20-22]. Concerns have been raised about the use of catecholamines in the treatment of septic shock [10].

\section{Beta Blockers, Sepsis, and the Immune System: Nonhuman Studies}

There has been longstanding interest in beta blockade and sepsis. As long ago as 1969, Berk et al. [23] used propranolol in a lipopolysaccharide (LPS) dog model and found that the beta blocker significantly improved survival when it was started 60 minutes after LPS insult. Propranolol also prevented hypotension and reduced fluid requirements. More recently, selective beta-1 blocking by esmolol decreased circulating TNF-alpha and IL-1beta concentrations in septic rats [24], reducing heart rate and blood pressure. Esmolol also protected LPS treated pigs from cardiovascular decline in a five hour model [25] so that in esmolol-treated animals the cardiac index had decreased by $9 \%$ after 3 hours and by only $2 \%$ at the end of the study; in controls the cardiac index had reduced by $14 \%$ and by $27 \%$, respectively $(P=0.870)$. This was despite a decrease in heart rate of $20 \%$ in the esmolol group and an increase in heart rate of $22 \%$ in controls $(P<0.001)$. Continuous infusion of esmolol initiated after septic insult improved survival at 5 days in a murine model [26] and noted an increase in the NFkappa B pathway. Similarly, landiolol, another selective beta-1-blocker, also decreased circulating levels of TNF-alpha, IL-6, and high mobility group box 1 in a rat model of endotoxin-induced sepsis [27]. Pretreatment with atenolol or metoprolol did not alter survival in a cecal ligation and puncture (CLP) rat model [28] given 2 hrs before CPL but the median time to death was increased by $33 \mathrm{hrs}$ in metoprolol-treated rats $(P=0.03)$. Metoprolol pretreatment reduced hepatic expression of proinflammatory cytokines and lowered plasma IL-6 (both $P<0.05$ ). Myocardial protein expression of IL-18 and monocyte chemoattractant protein1 , key mediators of cardiac dysfunction in sepsis, were also reduced $(P<0.05)$. In another study, atenolol had an antiinflammatory effect by increasing IL-10 but had no effect on TNF-alpha or L-6 concentrations in an ovine model with $E$. coli septicaemia [29].

Animal studies of the use of beta-agonists and beta blockade do not, however, give consistent and predictable results. Catecholamines have been demonstrated to reduce isolated human neutrophil function by decreasing free radical production, [30]. Early use of catecholamines at the same time as exposure of cells to LPS leads to the downregulation of synthesis of proinflammatory cytokines such as TNFalpha, IL-6, and IL-1 [31-33], and upregulates synthesis of antiinflammatory cytokines (e.g., IL-10) [32, 33]. Schmitz et al. [34] demonstrated an increased mortality in mice treated with propranolol undergoing CLP when beta blocker treatment was started at the time of surgery. Authors [6] have speculated that initial fluid resuscitation was impaired and that the nonselective nature of propranolol reduced the cardioprotection proffered by beta blockers. This does not entirely explain all findings and ignores previous animal models [23] reporting benefit with propranolol.

Lang and colleagues [35] found that beta blockade exacerbated a sepsis-induced response in the presence of propranolol. They reported an increase in muscle IL-6 and TNFalpha mRNA but did not alter the increment in IL-1alpha. Furthermore in another study [36], epinephrine infusion did not increase mortality at 48 hours in a CLP model in mice but induced alterations of splenocyte apoptosis, splenocyte proliferation, and IL-2 release. Subsequent treatment with propranolol augmented the epinephrine-induced increase of splenocyte apoptosis, did not affect the decrease of splenocyte proliferation and IL-2 release, augmented the release of IL- 6 , and antagonized the mobilization of natural killer cells. There 
was also a significant increase in mortality in propranolol treated animals.

\section{Clinical Experience with Beta-Agonism}

Studies emerged in the mid-1980s suggesting that patients treated with dobutamine and who could improve their cardiovascular performance had a better survival than those who did not $[37,38]$. In particular, Shoemaker's often quoted paper [38] suggested that mortality from a protocol-guided resuscitation regimen could reduce mortality from $23 \%$ in the control group to $4 \%$ in surgical patients managed with a pulmonary artery catheter and the addition of dobutamine to achieve therapeutic goals that were supranormal values for cardiac output $\left(>4.5 \mathrm{~L} / \mathrm{min} \cdot \mathrm{m}^{2}\right), \mathrm{DO} 2,\left(>600 \mathrm{~mL} / \mathrm{min} \cdot \mathrm{m}^{2}\right)$, and $\mathrm{VO} 2\left(>170 \mathrm{~mL} / \mathrm{min} \cdot \mathrm{m}^{2}\right)$. A subsequent larger resuscitation study [39] also demonstrated improvements in patients admitted through the emergency department and resuscitated according to a CVP based protocol. Rivers' paper [39] now forms the basis for fluid resuscitation in the Surviving Sepsis Campaign Guidelines for the management of severe sepsis and septic shock [9].

Most intensivists agree that the act of starting dobutamine is not the intervention that improves survival but it is the ability to achieve supernormal physiological status that selects out survivors. Shoemaker's study was followed shortly after by a study from the UK [40] which failed to demonstrate improved mortality in patients with established sepsis. Indeed, their results suggested that aggressive efforts to increase oxygen consumption could have been detrimental as the in-hospital mortality was lower in the control group (34 percent) than in the treatment group (54 percent) $(P=$ $0.04 ; 95$ percent confidence interval, 0.9 to 39.1 percent). One important difference, as shall be discussed later, is the timing of intervention. Hayes's paper [40] specifically intervened on patients who had been admitted to ICU and not those admitted from the emergency department.

There were a number of small studies towards the end of the last century that were summarised [41] as "Insufficient evidence exists to support goal-directed therapy with vasopressors and inotropes in the treatment of sepsis syndrome." A later analysis by the Cochrane collaboration [42] concluded "Probably the choice of vasopressors in patients with shock does not influence the outcome...." In the days following Shoemaker's groundbreaking findings, it was therefore assumed that the use of beta agonism was appropriate for the management of sepsis despite the fact that Shoemaker studied patients in the perioperative period. There remain no wellconducted randomised trials comparing beta-agonism with placebo.

Although not directly studying sepsis, the Beta Agonist Lung injury Trial 2 (BALTI-2) [43] was a multicentre randomized controlled trial comparing the selective beta2 agonist salbutamol with placebo $(0.9 \%$ saline) in patients with Acute Respiratory Distress Syndrome (ARDS). Approximately $25 \%$ of the cases included in the trial had ARDS as a result of sepsis and almost $50 \%$ as a result of pneumonia. The study was stopped early (after recruitment of 326 of a planned 1334 patients) due to increased mortality in the treatment arm (10.9\% (95\% CI $1.0 \%$ to $20.4 \%$ ) absolute increase (34.2\% versus $23.3 \%$ ) in 28 -day mortality). $14.2 \%$ of patients in the salbutamol arm (versus 1.2 in the placebo) had the beta agonist stopped due to tachycardia (HR > $140 \mathrm{bpm}$ ) with $8.6 \%$ having treatment withdrawn due to arrhythmias.

A recent study has suggested that vasopressin used as a norepinephrine-sparing agent may improve outcomes from less severe septic shock. The overall conclusion of the Vasopressin versus Norepinephrine Infusion in Patients with Septic Shock (VASST) [44] study was that there was no mortality benefit by use of less norepinephrine. However, in the prospectively defined stratum of less severe septic shock, the mortality rate was lower in the vasopressin group than in the norepinephrine group at 28 days (26.5\% versus $35.7 \%$, $P=0.05)$; there continues to be a concern that sympathetic agonism is detrimental to at least some patients with septic shock.

\section{What Is the Clinical Evidence for Beta Blockers in the Noncardiac Critically Ill Patient?}

The normal myocardial expression of beta- 1 and beta- 2 adrenoceptors is in the approximate ratio of 3 to 1 . In severe heart failure, this changes to 3 to 2 [45]. Sympathetic stimulation causes post synaptic noradrenergic beta-1 receptor stimulation whereas beta-2 adrenoceptors, located at extra junctional as well as post synaptic sites, are stimulated by circulating catecholamines [46]. This relative downregulation of beta- 1 adrenoceptors may well be a protective mechanism designed to shield the heart from a high sympathetic drive. Iatrogenically mimicking this with the use of beta blockers may protect the heart from sympathetic overstimulation in septic patients. An elevated heart rate is associated with worse outcomes in septic patients [13]. As the severity of sepsis increases (SIRS to sepsis to septic shock), there is reduced heart rate variability and sympathetic activation worsens [47]. Excessive tachycardia seen in many septic patients reduces diastolic filling time, increases myocardial oxygen consumption, and may result in tachycardia induced cardiomyopathy [48].

Drugs that are antagonists to beta receptors have different affinities for beta-1 and beta-2 subtypes. Esmolol has a selectivity of beta- 1 to beta- 2 ratio of 33 and a half-life $(t 1 / 2)=$ 9.19 min [49] with landiolol having a greater affinity for the beta- 1 receptor of beta- 1 to beta- 2 ratio 255 and $t 1 / 2=$ $3.96 \mathrm{~min}$ [50]. In comparison, propranolol is $74-380$ less selective for beta-1 when compared with landiolol and 39-263 when compared with esmolol [51]. Using recombinant cells expressing beta-1 and beta-2 receptors, Smith and Teitler [52] determined that bisoprolol was 19 times more selective for the beta- 1 receptor and metoprolol 6 times more selective for the beta-1 receptor when compared with propranolol. It is hardly surprising then that in combination with the proportions of beta-1 to beta- 2 receptors changing in health and disease, the response to beta blockade will vary depending upon how established sepsis is and which drug is selected.

The link between the adrenergic and immune systems requires further investigation: for example, beta blockade 
has also been shown to reduce proinflammatory cytokines in heart failure [53], critically ill trauma patients [54], and appears to have a beneficial effect on the T helper 1/T helper 2 ratio [55]. In a small trial including 55 critically injured patients at increased risk for heart disease, administration of metoprolol or esmolol decreased serum interleukin- (IL-) 6 levels [56]. A heart rate above $95 \mathrm{bpm}$ is associated with major cardiac events in critically ill patients [22] but this is likely to be the result of effects on many pathways rather than solely improving myocardial oxygen utilisation.

Christensen and colleagues [57] performed a retrospective study in their ICU on 8087 patients over 6 years. In this case-matched study of 3112 patients (1556 users of beta blockers and 1556 nonusers), the 30 -day mortality was $25.7 \%$ among beta blocker users and 31.4\% among nonusers (OR 0.74 ( $95 \%$ CI: 0.63 to 0.87 )). The OR was 0.69 (95\% CI: 0.54 to 0.88 ) for surgical ICU patients and 0.71 (95\% CI: 0.51 to 0.98 ) for medical ICU patients. The OR was 0.99 (95\% CI: 0.67 to 1.47 ) among users of nonselective beta blockers, and 0.70 (95\% CI: 0.58 to 0.83 ) among users of cardioselective beta blockers.

Herndon and colleagues successfully used propranolol to reduce heart rate by $20 \%$ in burned septic children and demonstrated attenuated hypermetabolism and reversal of muscle-protein catabolism both in the short-term [58] and over 12 months [59]. A retrospective analysis [60] examining 43 patients either already treated with (21 patients) or commencing beta blockers for other conditions in hospital (such as hypertension and tachyarrhythmias, 22 patients) suggested on multivariate analysis that pretreatment with beta blockers was associated with a significant decrease in fatal outcome and healing time.

Esmolol has undergone an evaluation of its safety in septic patients in several small studies; two from Europe [61, 62] and the authors understand that two case series have reported the safe use of esmolol in septic patients in China $[63,64]$. In his follow-on study, Morelli [1] studied patients admitted to his hospital with septic shock who had received haemodynamic optimisation of fluid resuscitation and vasopressor administration to maintain a MAP $\geq 65 \mathrm{mmhg}$, CVP $\geq 8 \mathrm{~cm}$ $\mathrm{H}_{2} 0$, pulmonary artery wedge pressure (PAWP) $\geq 12 \mathrm{mmHg}$, and mixed venous saturations $(\mathrm{SvO} 2)>65 \%$. The primary outcome was reduction in heart rate within $24 \mathrm{hrs}$ which was achieved. Surprisingly however, norepinephrine and resuscitative fluid volume requirements were also reduced (median area under the curve (AUC) for norepinephrine of $-0.11 \mu \mathrm{g} / \mathrm{kg} / \mathrm{min}$ versus $-0.01 \mu \mathrm{g} / \mathrm{kg} / \mathrm{min}, P=0.003$. Median AUC for fluid $3975 \mathrm{~mL} / 24 \mathrm{hrs}$ versus $4425 \mathrm{~mL} / 24 \mathrm{hr}$, $P<0.001)$. Stroke volume, systemic vascular resistance, and left ventricular stroke work indices were all higher in the esmolol group whilst maintaining $\mathrm{MAP} \geq 65 \mathrm{mmHg}$. In spite of a predictably reduced oxygen delivery (Do2) (median AUC $-100 \mathrm{~mL} / \mathrm{min} / \mathrm{m}^{2}$ versus $\left.-32 \mathrm{~mL} / \mathrm{min} / \mathrm{m}^{2}, P<0.001\right)$, lactate, base excess, and arterial $\mathrm{pH}$ were all higher in the esmolol group. Although not powered to discover a mortality outcome, the authors also reported a substantial reduction in mortality with a twenty-eight-day mortality of $49.4 \%$ in the esmolol group and $80.5 \%$ in the control group.

\section{Conclusion}

Recent improvement in the mortality from sepsis has largely come from increased awareness, early management, and rationalisation of existing supportive measures. Despite extensive research, the literature is littered with numerous therapies that brought initial hopes of disease modulation only to be found not to make patients survive longer and, in some cases, increased mortality. Beta blockers have been shown to have immunomodulatory actions in addition to their cardiovascular effect and a phase II clinical trial [1] has suggested that beta blockers may have an important role in the treatment of the patient with septic shock. This study has raised questions and stimulated interest into why, when, and in whom beta blockers are beneficial. In particular, it raises the immediate question: (1) is the effect specifically and only seen with esmolol or would other beta-1 specific antagonists also prove beneficial? (2) When should beta blockers be started? (3) Does the persistence of tachycardia at 24 hours following resuscitation and presentation with septic shock select out a group of patients genetically predisposed to do worse in sepsis and benefit from beta blockade?

Morelli's findings are tantalising to ICU physicians, some of whom are already using beta blockade in patients with established sepsis. Whether their findings are borne out by larger, multicentre studies remains to be seen.

\section{Conflict of Interests}

The authors declare that there is no conflict of interests regarding the publication of this paper.

\section{References}

[1] A. Morelli, C. Ertmer, M. Westphal et al., "Effect of heart rate control with esmolol on hemodynamic and clinical outcomes in patients with septic shock: a randomized clinical trial," The Journal of the American Medical Association, vol. 310, no. 16, pp. 1683-1691, 2013.

[2] I. J. Elenkov, R. L. Wilder, G. P. Chrousos, and E. S. Vizi, “The sympathetic nerve-an integrative interface between two supersystems: the brain and the immune system," Pharmacological Reviews, vol. 52, no. 4, pp. 595-638, 2000.

[3] A. Rudiger and M. Singer, "Mechanisms of sepsis-induced cardiac dysfunction," Critical Care Medicine, vol. 35, pp. 15991608, 2007.

[4] K. Muthu, J. Deng, F. Romano et al., “Thermal injury and sepsis modulates beta-adrenergic receptors and cAMP responses in monocyte-committed bone marrow cells," Journal of Neuroimmunology, vol. 165, no. 1-2, pp. 129-138, 2005.

[5] M. J. Cohen, R. Shankar, J. Stevenson, R. Fernandez, R. L. Gamelli, and S. B. Jones, "Bone marrow norepinephrine mediates development of functionally different macrophages after thermal injury and sepsis," Annals of Surgery, vol. 240, no. 1, pp. 132-141, 2004.

[6] A. Rudiger, "Beta-block the septic heart," Critical Care Medicine, vol. 38, no. 10, pp. S608-S612, 2010.

[7] D. Annane, E. Bellissant, and J. Cavaillon, "Septic shock," The Lancet, vol. 365, no. 9453, pp. 63-78, 2005. 
[8] C. R. Benedict and J. A. Rose, "Arterial norepinephrine changes in patients with septic shock," Circulatory Shock, vol. 38, no. 3, pp. 165-172, 1992.

[9] R. P. Dellinger, M. M. Levy, A. Rhodes et al., "Surviving sepsis campaign: international guidelines for management of severe sepsis and septic shock, 2012," Intensive Care Medicine, vol. 39, no. 2, pp. 165-228, 2013.

[10] M. Singer, "Catecholamine treatment for shock-equally good or bad?" The Lancet, vol. 370, no. 9588, pp. 636-637, 2007.

[11] C. H. Lang, "Sepsis-induced insulin resistance in rats is mediated by a $\beta$-adrenergic mechanism," The American Journal of Physiology: Endocrinology and Metabolism, vol. 263, no. 4, pp. E703-E711, 1992.

[12] D. M. Hargrove, G. J. Bagby, C. H. Lang, and J. J. Spitzer, "Adrenergic blockade prevents endotoxin-induced increases in glucose metabolism," American Journal of Physiology, vol. 255, no. 5, part 1, pp. E629-E635, 1988.

[13] M. M. Parker, J. H. Shelhamer, C. Natanson, D. W. Alling, and J. E. Parrillo, "Serial cardiovascular variables in survivors and nonsurvivors of human septic shock: heart rate as an early predictor of prognosis," Critical Care Medicine, vol. 15, no. 10, pp. 923-929, 1987.

[14] D. G. Evans, A. A. Miles, and J. S. Niven, "The enhancement of bacterial infections by adrenaline," British Journal of Experimental Pathology, vol. 29, no. 1, pp. 20-39, 1948.

[15] M. Lyte, P. P. E. Freestone, C. P. Neal et al., "Stimulation of Staphylococcus epidermidis growth and biofilm formation by catecholamine inotropes," The Lancet, vol. 361, no. 9352, pp. 130$135,2003$.

[16] P. P. E. Freestone, R. D. Haigh, and M. Lyte, "Specificity of catecholamine-induced growth in Escherichia coli O157:H7, Salmonella enterica and Yersinia enterocolitica," FEMS Microbiology Letters, vol. 269, no. 2, pp. 221-228, 2007.

[17] P. P. E. Freestone, M. Lyte, C. P. Neal, A. F. Maggs, R. D. Haigh, and P. H. Williams, "The mammalian neuroendocrine hormone norepinephrine supplies iron for bacterial growth in the presence of transferrin or lactoferrin," Journal of Bacteriology, vol. 182, no. 21, pp. 6091-6098, 2000.

[18] P. Y. Hahn, P. Wang, S. M. Tait, Z. F. Ba, S. S. Reich, and I. H. Chaudry, "Sustained elevation in circulating catecholamine levels during polymicrobial sepsis," Shock, vol. 4, no. 4, pp. 269273, 1995.

[19] J. Boldt, T. Menges, D. Kuhn, C. Diridis, and G. Hempelmann, "Alterations in circulating vasoactive substances in the critically ill-a comparison between survivors and non-survivors," Intensive Care Medicine, vol. 21, no. 3, pp. 218-225, 1995.

[20] M. W. Dünser and W. R. Hasibeder, "Sympathetic overstimulation during critical illness: adverse effects of adrenergic stress," Journal of Intensive Care Medicine, vol. 24, no. 5, pp. 293-316, 2009.

[21] C. A. Schmittinger, C. Torgersen, G. Luckner, D. C. H. Schröder, I. Lorenz, and M. W. Dünser, "Adverse cardiac events during catecholamine vasopressor therapy: a prospective observational study," Intensive Care Medicine, vol. 38, no. 6, pp. 950-958, 2012.

[22] O. Sander, I. D. Welters, P. Foëx, and J. W. Sear, "Impact of prolonged elevated heart rate on incidence of major cardiac events in critically ill patients with a high risk of cardiac complications," Critical Care Medicine, vol. 33, no. 1, pp. 81-88, 2005.

[23] J. L. Berk, J. F. Hagen, W. H. Beyer, M. J. Gerber, and G. R. Dochat, "The treatment of endotoxin shock by beta adrenergic blockade," Annals of Surgery, vol. 169, no. 1, pp. 74-81, 1969.
[24] T. Suzuki, H. Morisaki, R. Serita et al., "Infusion of the $\beta$ adrenergic blocker esmolol attenuates myocardial dysfunction in septic rat," Critical Care Medicine, vol. 33, no. 10, pp. 22942301, 2005.

[25] J. Aboab, V. Sebille, M. Jourdain et al., "Effects of esmolol on systemic and pulmonary hemodynamics and on oxygenation in pigs with hypodynamic endotoxin shock," Intensive Care Medicine, vol. 37, no. 8, pp. 1344-1351, 2011.

[26] I. Ibrahim-Zada, P. Rhee, C. T. Gomez, J. Weller, and R. S. Friese, "Inhibition of sepsis-induced inflammatory response by $\beta 1$-adrenergic antagonists," Journal of Trauma and Acute Care Surgery, vol. 76, no. 2, pp. 320-328, 2014.

[27] S. Hagiwara, H. Iwasaka, H. Maeda, and T. Noguchi, "Landiolol, an ultrashort-acting $\beta 1$-adrenoceptor antagonist, has protective effects in an lps-induced systemic inflammation model," Shock, vol. 31, no. 5, pp. 515-520, 2009.

[28] G. L. Ackland, S. T. Yao, A. Rudiger et al., "Cardioprotection, attenuated systemic inflammation, and survival benefit of $\beta 1$ adrenoceptor blockade in severe sepsis in rats," Critical Care Medicine, vol. 38, no. 2, pp. 388-394, 2010.

[29] P. Calzavacca, Y. R. Lankadeva, S. R. Bailey, M. Bailey, R. Bellomo, and C. N. May, "Effects of selective betal-adrenoceptor blockade on cardiovascular and renal function and circulating cytokines in ovine hyperdynamic sepsis," Critical Care, vol. 18, article 610, 2014.

[30] M. Weiss, E. M. Schneider, J. Tarnow et al., "Is inhibition of oxygen radical production of neutrophils by sympathomimetics mediated via beta-2 adrenoceptors?" Journal of Pharmacology and Experimental Therapeutics, vol. 278, no. 3, pp. 1105-1113, 1996.

[31] A. Severn, N. T. Rapson, C. A. Hunter, and F. Y. Liew, "Regulation of tumor necrosis factor production by adrenaline and beta-adrenergic agonists," The Journal of Immunology, vol. 148, no. 11, pp. 3441-3445, 1992.

[32] K. Muthu, J. Deng, R. Gamelli, R. Shankar, and S. B. Jones, "Adrenergic modulation of cytokine release in bone marrow progenitor-derived macrophage following polymicrobial sepsis," Journal of Neuroimmunology, vol. 158, no. 1-2, pp. 50-57, 2005.

[33] J. Deng, K. Muthu, R. Gamelli, R. Shankar, and S. B. Jones, "Adrenergic modulation of splenic macrophage cytokine release in polymicrobial sepsis," The American Journal of PhysiologyCell Physiology, vol. 287, no. 3, pp. C730-C736, 2004.

[34] D. Schmitz, K. Wilsenack, S. Lendemanns, M. Schedlowski, and R. Oberbeck, " $\beta$-Adrenergic blockade during systemic inflammation: impact on cellular immune functions and survival in a murine model of sepsis," Resuscitation, vol. 72, no. 2, pp. 286294, 2007.

[35] C. H. Lang, G. Nystrom, and R. A. Frost, "Beta-adrenergic blockade exacerbates sepsis-induced changes in tumor necrosis factor alpha and interleukin-6 in skeletal muscle and is associated with impaired translation initiation," The Journal of Trauma, vol. 64, no. 2, pp. 477-486, 2008.

[36] R. Oberbeck, D. Schmitz, K. Wilsenack et al., "Adrenergic modulation of survival and cellular immune functions during polymicrobial sepsis," NeuroImmunoModulation, vol. 11, no. 4, pp. 214-223, 2004.

[37] R. D. Bland, W. C. Shoemaker, E. Abraham, and J. C. Cobo, "Hemodynamic and oxygen transport patterns in surviving and nonsurviving postoperative patients," Critical Care Medicine, vol. 13, no. 2, pp. 85-90, 1985. 
[38] W. C. Shoemaker, P. L. Appel, H. B. Kram, K. Waxman, and T.-S. Lee, "Prospective trial of supranormal values of survivors as therapeutic goals in high-risk surgical patients," Chest, vol. 94, no. 6, pp. 1176-1186, 1988.

[39] E. Rivers, B. Nguyen, S. Havstad et al., "Early goal-directed therapy in the treatment of severe sepsis and septic shock," The New England Journal of Medicine, vol. 345, no. 19, pp. 1368-1377, 2001.

[40] M. A. Hayes, A. C. Timmins, E. H. S. Yau, M. Palazzo, C. J. Hinds, and D. Watson, "Elevation of systemic oxygen delivery in the treatment of critically ill patients," The New England Journal of Medicine, vol. 330, no. 24, pp. 1717-1722, 1994.

[41] M. I. Rudis, M. A. Basha, and B. J. Zarowitz, "Is it time to reposition vasopressors and inotropes in sepsis?" Critical Care Medicine, vol. 24, no. 3, pp. 525-537, 1996.

[42] C. Havel, J. Arrich, H. Losert, G. Gamper, M. Müllner, and H. Herkner, "Vasopressors for hypotensive shock," Cochrane Database of Systematic Reviews, vol. 5, Article ID CD003709, 2011.

[43] S. Gates, G. D. Perkins, S. E. Lamb et al., "Beta-Agonist Lung injury TrIal-2 (BALTI-2): a multicentre, randomised, doubleblind, placebo-controlled trial and economic evaluation of intravenous infusion of salbutamol versus placebo in patients with acute respiratory distress syndrome," Health Technology Assessment, vol. 17, no. 38, pp. 1-87, 2013.

[44] J. A. Russell, K. R. Walley, J. Singer et al., "Vasopressin versus norepinephrine infusion in patients with septic shock," The New England Journal of Medicine, vol. 358, no. 9, pp. 877-887, 2008.

[45] M. R. Bristow, R. Ginsburg, V. Umans et al., "Beta 1- and beta 2 -adrenergic-receptor subpopulations in nonfailing and failing human ventricular myocardium: coupling of both receptor subtypes to muscle contraction and selective beta 1-receptor down-regulation in heart failure," Circulation Research, vol. 59, no. 3, pp. 297-309, 1986.

[46] D. B. Barnett, "Myocardial $\beta$-adrenoceptor function and regulation in heart failure: implications for therapy," British Journal of Clinical Pharmacology, vol. 27, no. 5, pp. 527-537, 1989.

[47] D. Annane, F. Trabold, T. Sharshar et al., "Inappropriate sympathetic activation at onset of septic shock: a spectral analysis approach," American Journal of Respiratory and Critical Care Medicine, vol. 160, no. 2, pp. 458-465, 1999.

[48] F. J. Romero-Bermejo, M. Ruiz-Bailen, J. Gil-Cebrian, and M. J. Huertos-Ranchal, "Sepsis-induced cardiomyopathy," Current Cardiology Reviews, vol. 7, no. 3, pp. 163-183, 2011.

[49] C. Y. Sum, A. Yacobi, R. Kartzinel, H. Stampfli, C. S. Davis, and C.-M. Lai, "Kinetics of esmolol, an ultra-short-acting beta blocker, and of its major metabolite," Clinical Pharmacology and Therapeutics, vol. 34, no. 4, pp. 427-434, 1983.

[50] S. Iguchi, H. Iwamura, M. Nishizaki et al., "Development of a highly cardioselective ultra short-acting $\beta$-blocker, ONO-1101," Chemical and Pharmaceutical Bulletin, vol. 40, no. 6, pp. 14621469, 1992.

[51] H. Tsuchiya and M. Mizogami, "Characteristic interactivity of landiolol, an ultra-short-acting highly selective $\beta_{1}$-blocker, with biomimetic membranes: comparisons with $\beta_{1}$-selective esmolol and non-selective propranolol and alprenolol," Frontiers in Pharmacology, Article ID Article 150, 2013.

[52] C. Smith and M. Teitler, "Beta-blocker selectivity at cloned human betal- and beta2-adrenergic receptors," Cardiovascular Drugs and Therapy, vol. 13, no. 2, pp. 123-126, 1999.

[53] T. Ohtsuka, M. Hamada, G. Hiasa et al., "Effect of beta-blockers on circulating levels of inflammatory and anti-inflammatory cytokines in patients with dilated cardiomyopathy," Journal of the American College of Cardiology, vol. 37, no. 2, pp. 412-417, 2001.

[54] R. S. Friese, R. Barber, D. McBride, J. Bender, and L. M. Gentilello, "Could beta blockade improve outcome after injury by modulating inflammatory profiles?" Journal of Trauma, vol. 64, no. 4, pp. 1061-1068, 2008.

[55] X. Tian, L. Zhang, Y. Hou et al., "Effects of cAMP and $\beta$ adrenergic receptor antagonists on the function of peripheral T helper lymphocytes in patients with heart failure," NeuroImmunoModulation, vol. 18, no. 2, pp. 73-78, 2010.

[56] M. Bergmann, A. Gornikiewicz, T. Sautner et al., "Attenuation of catecholamine-induced immunosuppression in whole blood from patients with sepsis," Shock, vol. 12, no. 6, pp. 421-427, 1999.

[57] S. Christensen, M. B. Johansen, E. Tønnesen et al., "Preadmission beta-blocker use and 30-day mortality among patients in intensive care: a cohort study," Critical Care, vol. 15, no. 2, article R87, 2011.

[58] D. N. Herndon, D. W. Hart, S. E. Wolf, D. L. Chinkes, and R. R. Wolfe, "Reversal of catabolism by beta-blockade after severe burns," The New England Journal of Medicine, vol. 345, no. 17, pp. 1223-1229, 2001.

[59] D. N. Herndon, N. A. Rodriguez, E. C. Diaz et al., "Longterm propranolol use in severely burned pediatric patients: A randomized controlled study," Annals of Surgery, vol. 256, no. 3, pp. 402-411, 2012.

[60] S. Arbabi, E. M. Campion, M. R. Hemmila et al., "Beta-blocker use is associated with improved outcomes in adult trauma patients," Journal of Trauma, vol. 62, no. 1, pp. 56-61, 2007.

[61] M. Balik, J. Rulisek, P. Leden et al., "Concomitant use of beta1 adrenoreceptor blocker and norepinephrine in patients with septic shock," Wiener Klinische Wochenschrift, vol. 124, no. 1516, pp. 552-556, 2012.

[62] A. Morelli, A. Donati, C. Ertmer et al., "Microvascular effects of heart rate control with esmolol in patients with septic shock: a pilot study," Critical Care Medicine, vol. 41, no. 9, pp. 2162-2168, 2013.

[63] J.-X. Chen, J. Sun, Y.-Y. Liu, and B.-H. Jia, "Effects of adrenergic beta-1 antagonists on hemodynamics of severe septic patients," Zhonghua Yi Xue Za Zhi, vol. 93, no. 16, pp. 1243-1246, 2013.

[64] S. Yang, Z. Liu, W. Yang et al., "Effects of the beta-blockers on cardiac protection and hemodynamics in patients with septic shock: a prospective study," Zhonghua Wei Zhong Bing Ji Jiu Yi Xue, vol. 26, pp. 714-717, 2014. 


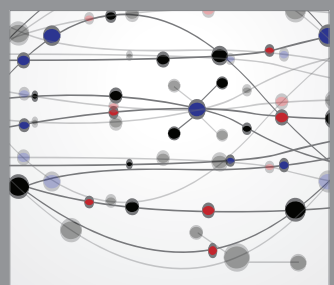

The Scientific World Journal
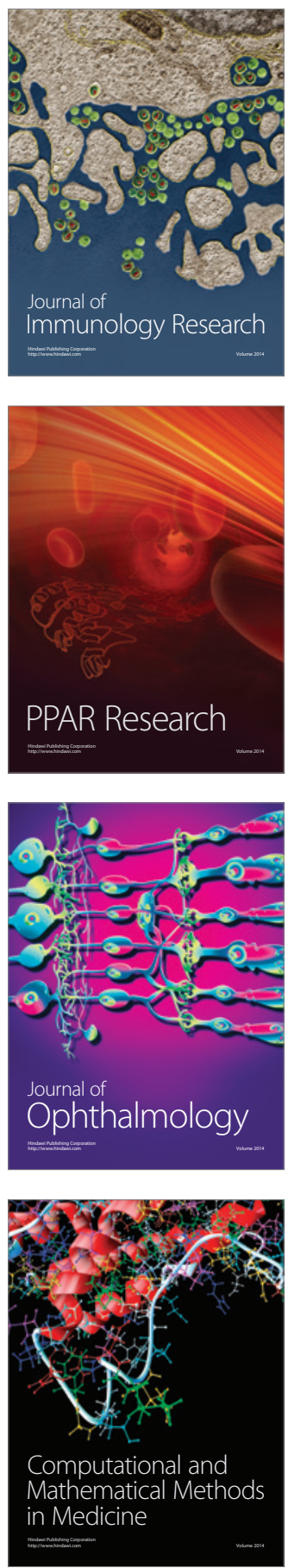

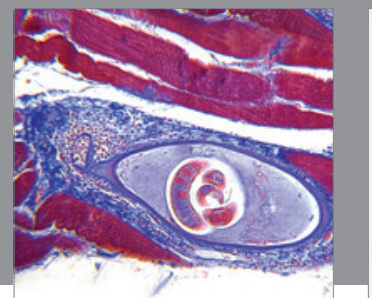

Gastroenterology

Research and Practice
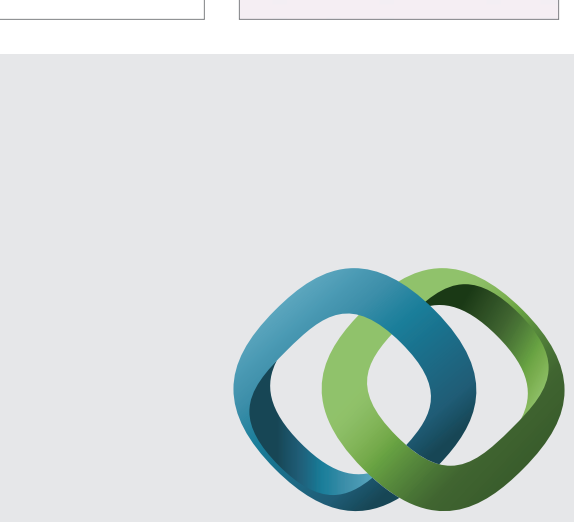

\section{Hindawi}

Submit your manuscripts at

http://www.hindawi.com
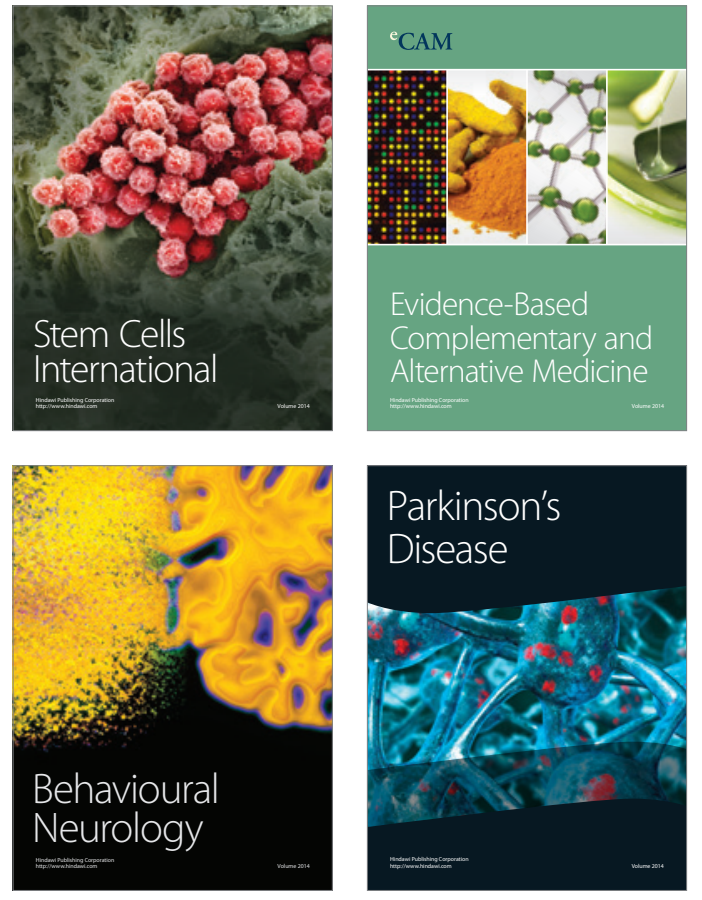
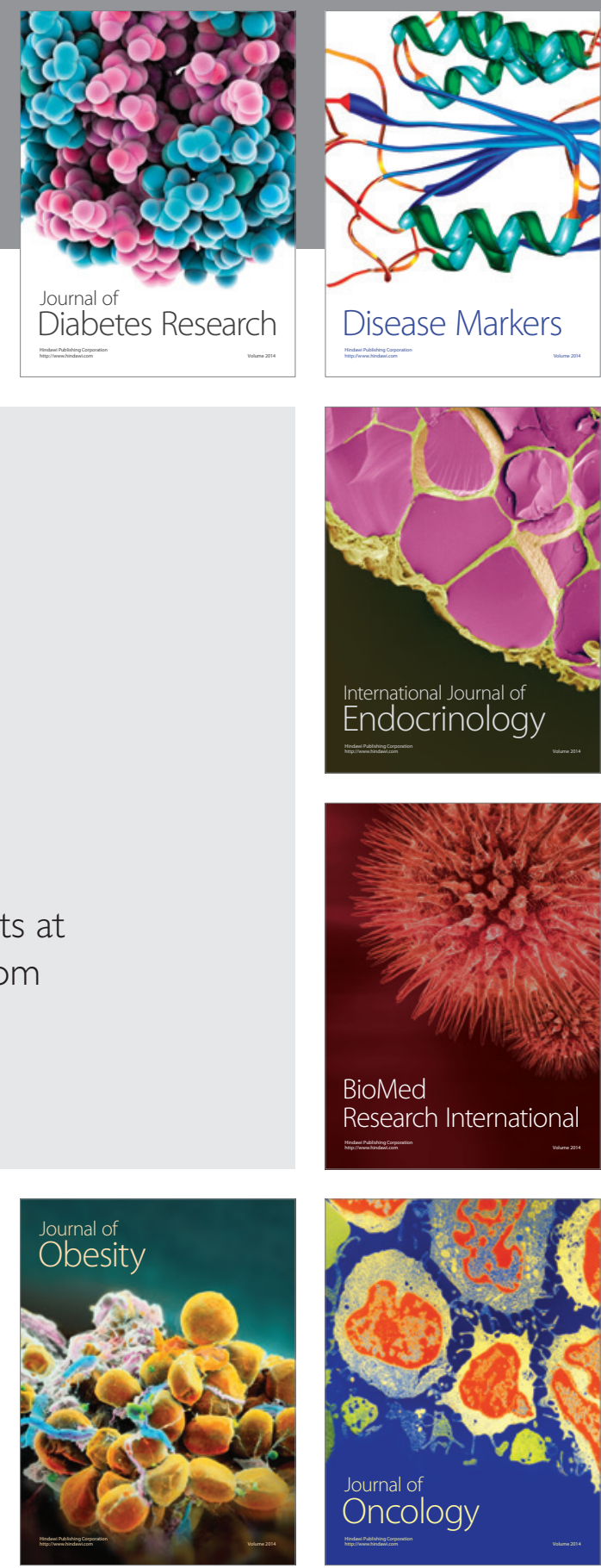

Disease Markers
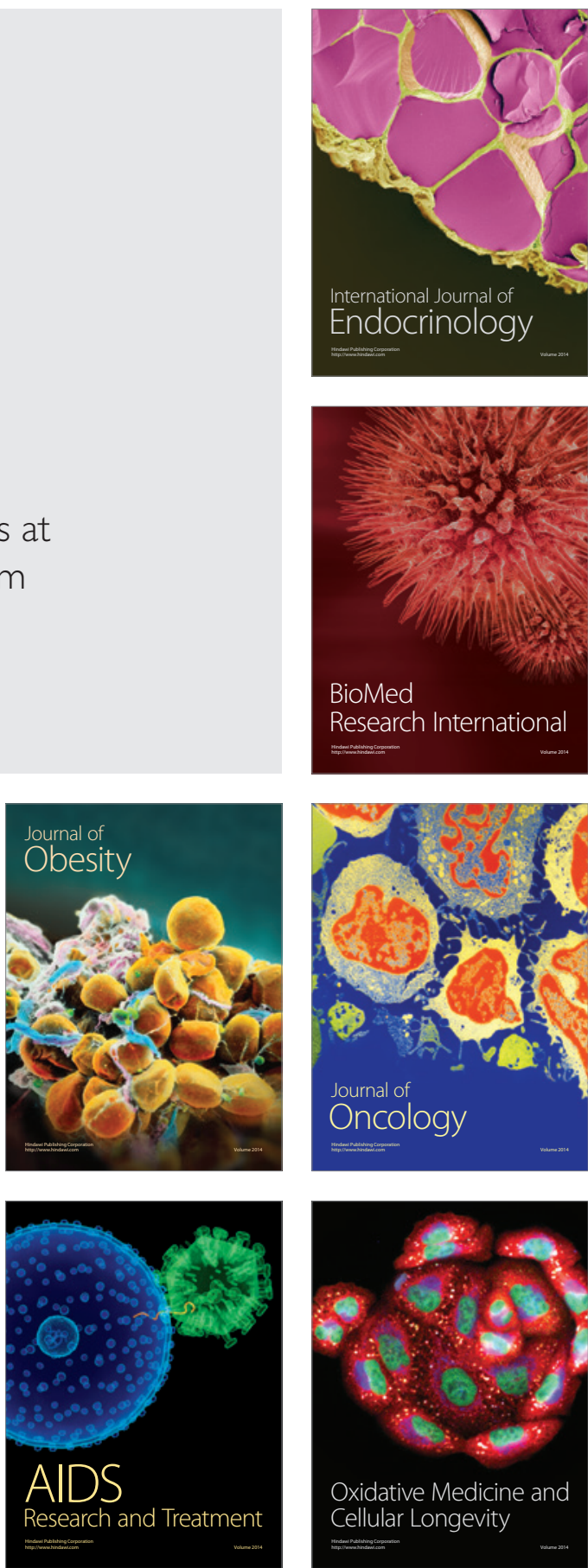